\title{
Investigating the Relationship between Low Serum Cholesterol and Suicide in Attempters with Depression
}

\author{
Nader Aghakhani ${ }^{1}$ (D), Behzad Boushehri ${ }^{2}$ (D), Abbas Zarei ${ }^{3}$ (D), Rasoul Roshani ${ }^{3}$ (D), Narges Nazimi ${ }^{4}$ (D) \\ Rozita Cheraghi $^{1}$, Hojjat Sayyadi ${ }^{5}$, Davoud Vahabzadeh ${ }^{*}$ (D)
}

1. Clinical Research Institute, Patient Safety Research Center, Urmia University of Medical Sciences, Urmia, Iran

2. Dept.of Forensic Medicine,School of Medicine, Taleghani Hospital, Urmia University of Medical Sciences, Urmia, Iran

3. Forensic Medicine Organization, West Azarbaijan Branch,Urmia,Iran

4. Forensic Medicine Organization,Tehran, Iran

5. Faculty of Health,Ilam University of Medical Sciences, Ilam, Iran

6. Dept. of Nutrition \& Biochemistry, Faculty of Medicine, Ilam University of Medical Sciences, Ilam, Iran

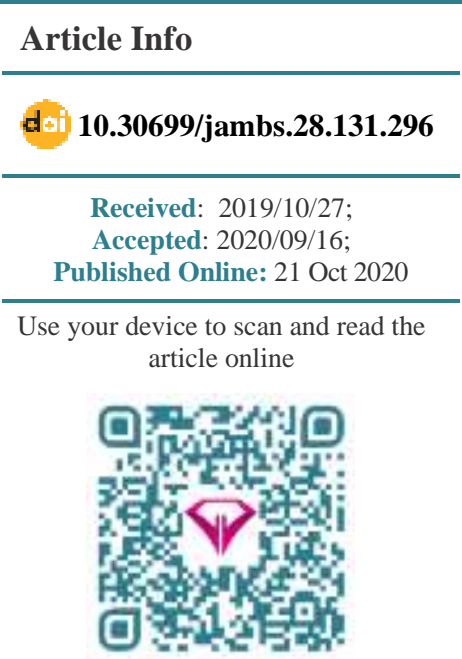

Corresponding Information: Davoud Vahabzadeh,

Dept. of Nutrition \& Biochemistry, Faculty of Medicine, Ilam University of Medical Sciences, Ilam, Iran E-Mail:

dvaha2003@yahoo.com

\section{ABSTRACT}

Background \& Objective: It has been postulated that depressed individuals with low total cholesterol levels may be more likely to die prematurely from suicide. This study aimed to examine the association between low serum cholesterol and suicide in depressed attempters.

Materials \& Methods: In this cross-sectional study, 180 suicide attempters, who met the inclusion criteria and were willing to participate in the study, were recruited in 2017. The data was collected using a demographic questionnaire and the Beck Depression Inventory-Second Edition (BDI-II). The blood cholesterol level was measured via an auto-analyzer.

Results: The mean age was $26.39 \pm 10.75$ years. The average cholesterol level in the moderate, severe, and serious depression groups was $151.30 \pm 35.23,145.89 \pm 36.32$, and $145.15 \pm 33.33$, respectively. The mean age was higher in the group with a higher depression level, though the difference was not significant $(P=0.06)$. The percentage of suicide attempts in single individuals was significantly higher $(P=0.02)$. The mean cholesterol level in the group with the highest level of depression was the lowest, but the difference was insignificant ( $\mathrm{r}=-.01, P=0.85)$. Only in females, the level of blood cholesterol showed a nearly significant difference between groups with different severities of depression $(P=0.05)$. Cholesterol had a significant correlation with suicide frequency $(P=0.008, \mathrm{r}=0.28)$.

Conclusion: Our results revealed no significant association between low serum cholesterol and suicide in attempters with depression; but low total serum cholesterol may be associated with depression and suicide in depressed subjects. Yet, more studies are required for verification of this causality.

\section{Introduction}

Suicide is a major public health concern. It accounts for $1-2 \%$ of global deaths and ranks the $17^{\text {th }}$ among the leading causes of mortality (1). According to the estimates of the World Health Organization (WHO), about 1.5 million individuals die due to suicide each year worldwide (2). Some studies have demonstrated the relationship between lower cholesterol levels and suicide attempts in patients admitted to hospitals following suicide attempts compared to patients with no attempt history (3). However, several case-control studies reported no relationship between cholesterol levels and recent suicide attempts (4).
It has been reported that some factors, including depression and hopelessness experiences or problems with social communication can increase the suicide risk; this reflects the risk of a suicide crisis, and thus requires immediate intervention (2). In Iran, the rate of suicide is lower than western countries; however, it is higher than its average in the Middle East region. The incidence of suicide in the western provinces of Iran has been reported to be higher than its total average in the country (5). The costs of suicide are not limited to the lives lost in the community, but also it imposes mental and physical stress on the family and society. Suicide occurs 
due to interactions of several different factors such as genetic and environmental issues, medical and social support, age, sex, level of education, marital and economic status, drinking behavior or smoking status, and mental and personality disorders (6-8).

Suicide attempt, as a situation in which a person has performed a life-threatening behavior with the intent of putting his/her life at risk, is the worst complication of depression as one of the four major diseases. According to the WHO, depression affects about 350 million individuals in the world (9). In individuals who have experienced a psychologically traumatic event, the risk factors for depression can include a family history, insufficient income, age, and sex (10). Moreover, it has been postulated that some metabolic parameters, such as the level of fasting plasma glucose, plasma levels of total cholesterol, triglycerides, high-density lipoprotein cholesterol (HDL-c), and low-density lipoprotein cholesterol (LDL-c) or neuropsychiatric conditions may be associated with some mental disorders, particularly depression $(3,11,12)$.

The mechanism that may link the level of serum lipids with suicidal activities is still unclear. It has been proposed that low peripheral cholesterol in individuals with psychiatric disorders may be accompanied by common regulatory mechanisms that can induce changes in specific synaptic lipid rafts which, in turn, reduce the level of serotonergic activities and finally lead to impulsivity and violent suicidal behaviors (3).

In addition, it has been assumed that low serum cholesterol levels can induce changes in the micro-viscosity and function of serotonin receptors and transporters in neural membranes. Moreover, some investigators have proposed the existence of an association between low serum cholesterol levels and serotonin transporter polymorphisms $(3,13)$.

Considering the increasing rate of suicide in recent years and the tendency of studies to identify its leading causes (e.g. low serum cholesterol), this study was carried out to examine the association between serum cholesterol levels and depression in suicidal patients visiting the toxicology department of a university hospital West Azarbaijan province, Iran.

\section{Materials and Methods}

\section{Participants and Clinical Evaluation}

This cross-sectional study was conducted on 180 participants who had committed suicide and then been admitted to an educational and treatment center in Urmia, Iran in the period between February and August 2017. The sampling method was the census procedure. Since most suicide cases in West Azerbaijan province are referred to toxicology department of Ayatollah Taleghani University Hospital in Urmia, after the admission of each patient to this department, his/her data were evaluated to deem his/her eligibility to participate in the study. The sample size was calculated based on the data from previous studies.

The inclusion criteria were: written consent to participate in the study, lack of a history of taking cholesterol-lowering drugs, lack of psychedelic drug abuse, no history of alcohol consumption, no history of cholesterol disorders, no history of mental disorders (based on medical documents or self-reports), no family history of high blood cholesterol, and no history of endocrine, cardiac, renal or liver diseases.

All the participants signed an informed consent letter. A demographic questionnaire was used to collect the following information: age, sex, occupation, educational level, marital status, place of residence, history of suicide attempt, and the reason for poisoning him/herself. Then, we administered the Beck Depression Inventory-Second Edition (BDI-II), which consisted of behavioral and cognitive dimensions with 21 self-report questions, whose validity and reliability have been evaluated in other studies on the Iranian sample population. Scores $<10$ were considered as normal, 1019 as mild, 20-29 as medium, 30-39 as severe, and >40 as serious depression.

Blood Sampling and Biochemical Assessments:

From all the participants, 5cc fasting blood samples were taken in the laboratory, and the cholesterol level was measured via an auto-analyzer (Model 200-747; Hitachi, Dubai). According to the American Heart Association (AHA) guidelines, total cholesterol levels $<200 \mathrm{mg} / \mathrm{dl}$, $200-239 \mathrm{mg} / \mathrm{dl}$, and $>240 \mathrm{mg} / \mathrm{dl}$ were considered as normal, borderline, and high, respectively (14).

\section{Ethical Considerations}

This project complied with the principles of the Helsinki Declaration regarding medical research studies in humans, following the allocation of a local research ethical code from Urmia University of Medical Sciences (IR.umsu.rec.1395.590)

\section{Statistical Analysis}

After collecting and coding the data, they were analyzed in SPSS version 20. Qualitative data were presented as number (percentage), and quantitative variables were reported as mean \pm SD. Correlation analysis was performed using Pearson correlation and Spearman rank correlation tests, whenever appropriate. Analyses of variance (ANOVA) and chi-squared tests were also performed. A P-value $<0.05$ was considered statistically significant.

\section{Results}

Among 180 studied suicidal individuals, the majority of them had moderate or higher levels of depression. About 25.6\% had a moderate depression level, 51.1\% severe depression, and $23.3 \%$ serious depression. The average depression score in the group with moderate, severe, and serious depression levels was 26.04 \pm 6.45 , $34.54 \pm 2.8$, and $47.29 \pm 4.38$, respectively. In the group with a higher depression level, the mean age was 
higher; but the difference was not significant $(P=0.06)$. The percentage of suicide attempts in single individuals was significantly higher than in married ones $(P=0.02)$. The highest number of hospital admission belonged to those with severe depression $(P=0.006)$. In this population, people with different levels of depression did not show any significant difference in terms of income $(P=0.2)$ and methods of committing suicide. Demographic characteristics are presented in Table 1 . Most of the suicide attempters were females (54.4\%) rather than males $(45.6 \%)$. As for the method of attempting suicide, the results showed that $52.2 \%$ of the attempters made a suicide attempt with drugs, $42.2 \%$ with poisoning, and $5.6 \%$ with other methods.

Table 1. Comparing patients' characteristics among three different depression categories based on qualitative variable

\begin{tabular}{|c|c|c|c|c|c|}
\hline Variable & Categories & $\begin{array}{c}\text { Moderate } \\
\text { Depression } \\
(\mathbf{N} / \%)\end{array}$ & $\begin{array}{c}\text { Severe } \\
\text { Depression } \\
(\mathbf{N} / \%)\end{array}$ & $\begin{array}{c}\text { Serious } \\
\text { Depression }(\mathbf{N} / \%)\end{array}$ & P-value ${ }^{*}$ \\
\hline \multirow{2}{*}{ Sex } & Male & $26(56.5)$ & $42(45.7)$ & $14(33.3)$ & \multirow{2}{*}{0.03} \\
\hline & Female & $20(43.5)$ & $50(54.3)$ & $28(66.7)$ & \\
\hline \multirow{2}{*}{ Marital Status } & Single & $34(73.9)$ & $46(50)$ & $22(52.4)$ & \multirow{2}{*}{0.007} \\
\hline & Married & $12(26.1)$ & $46(50)$ & $18(42.9)$ & \\
\hline \multirow{4}{*}{ Education } & Illiterate & $8(17.4)$ & $14(15.2)$ & $6(14.3)$ & \multirow{4}{*}{0.002} \\
\hline & High School & $20(43.5)$ & $54(58.7)$ & $10(23.8)$ & \\
\hline & Diploma & $12(26.1)$ & $20(21.7)$ & $16(38.1)$ & \\
\hline & Academic & $6(13)$ & $4(4.3)$ & $10(23.8)$ & \\
\hline \multirow{4}{*}{$\begin{array}{l}\text { Hospitalization due } \\
\text { to suicide }\end{array}$} & First time & $38(82.6)$ & $58(63.0)$ & $22(52.4)$ & \multirow{4}{*}{0.017} \\
\hline & Second time & $8(17.4)$ & $30(32.6)$ & $16(38.1)$ & \\
\hline & Third time & $0(0)$ & $4(4.3)$ & $2(4.8)$ & \\
\hline & Fifth time & $0(0)$ & $0(0)$ & $2(4.8)$ & \\
\hline \multirow{3}{*}{ Suicide Method } & Taking the drug & $18(39.1)$ & $50(54.3)$ & 26(61.9) & \multirow{3}{*}{0.208} \\
\hline & Poison ingestion & $26(56.5)$ & $36(39.1)$ & $14(33.3)$ & \\
\hline & Other ways & $2(4.3)$ & $6(6.5)$ & $2(4.8)$ & \\
\hline \multirow{3}{*}{$\begin{array}{l}\text { Monthly income } \\
\text { status }\end{array}$} & More than spend & $34(73.9)$ & $52(56.5)$ & $28(66.7)$ & \multirow{3}{*}{0.014} \\
\hline & Equal to spend & $8(17.4)$ & $38(41.3)$ & $14(33.3)$ & \\
\hline & Less than spend & $4(8.7)$ & $2(2.2)$ & $0(0)$ & \\
\hline \multirow{3}{*}{$\begin{array}{l}\text { Monthly income } \\
\text { status }\end{array}$} & More than spend & $34(73.9)$ & $52(56.5)$ & $28(66.7)$ & \multirow{3}{*}{0.014} \\
\hline & Equal to spend & $8(17.4)$ & $38(41.3)$ & $14(33.3)$ & \\
\hline & Less than spend & $4(8.7)$ & $2(2.2)$ & $0(0)$ & \\
\hline
\end{tabular}

${ }^{*}$ Chi-square test

The mean age of the participants was $26.39 \pm 10.78$ years. The survey also showed that $82 \%$ of suicide attempters belonged to the age group of 15-24 years. The mean cholesterol level in the group with the highest level of depression was the lowest (Figure 1A). The data showed that when the rate of depression increased, the levels of blood cholesterol decreased insignificantly $(\mathrm{r}=-.01, P=0.85)$. Meanwhile, there was no significant difference in cholesterol levels between different groups $(P=0.64)$ (Table 2).

In this survey, the mean depression intensity in females and males was $34.39 \pm 7.41$ and 36.14 \pm 9.6 , respectively. While the percentage of females with severe depression was $42.2 \%$, it was $24.4 \%$ for males. This measure had no significant association with education level, marital status, economic status, or sex. The level of blood cholesterol showed a nearly significant difference between groups with different severities of depression $(P=0.05)$ only in females. Nevertheless, depression intensity was significantly associated with suicide frequency. The mean cholesterol levels in different depression categories are presented in Table 2. 
Table 2. Comparing patients' characteristics among three different depression categories based on qualitative variables

\begin{tabular}{|c|c|c|c|c|}
\hline Variable & $\begin{array}{l}\text { Moderate } \\
\text { Depression } \\
(\text { Mean } \pm \text { SD })\end{array}$ & $\begin{array}{c}\text { Severe } \\
\text { Depression } \\
(\text { Mean } \pm \text { SD })\end{array}$ & $\begin{array}{c}\text { Serious } \\
\text { Depression (Mean } \\
\pm \text { SD) }\end{array}$ & P Value ${ }^{*}$ \\
\hline Age (year) & $25.22 \pm 10.67$ & $25.43 \pm 10.10$ & $29.76 \pm 11.9$ & 0.06 \\
\hline $\begin{array}{l}\text { Blood Cholesterol (F) } \\
(\mathrm{mg} / \mathrm{dl})\end{array}$ & $157.7 \pm 37.9$ & $138.16 \pm 28.8$ & $151.08 \pm 36.1$ & 0.05 \\
\hline $\begin{array}{l}\text { Blood Cholesterol (M) } \\
\text { (mg/dl) }\end{array}$ & $146.8 \pm 32.9$ & $155.10 \pm 42.18$ & $134.14 \pm 25.09$ & 0.17 \\
\hline
\end{tabular}

There was no correlation between cholesterol level and age, depression, suicide frequency, suicide method, suicide attempts, and the possibility of suicide. Besides, there was no relationship between the rate of depression and suicide attempts; however, this relationship was significant with the frequency of suicide $(P=0.008$, $\mathrm{r}=0.28)$. Figure 1-B displays the correlation between blood cholesterol levels and depression scores.
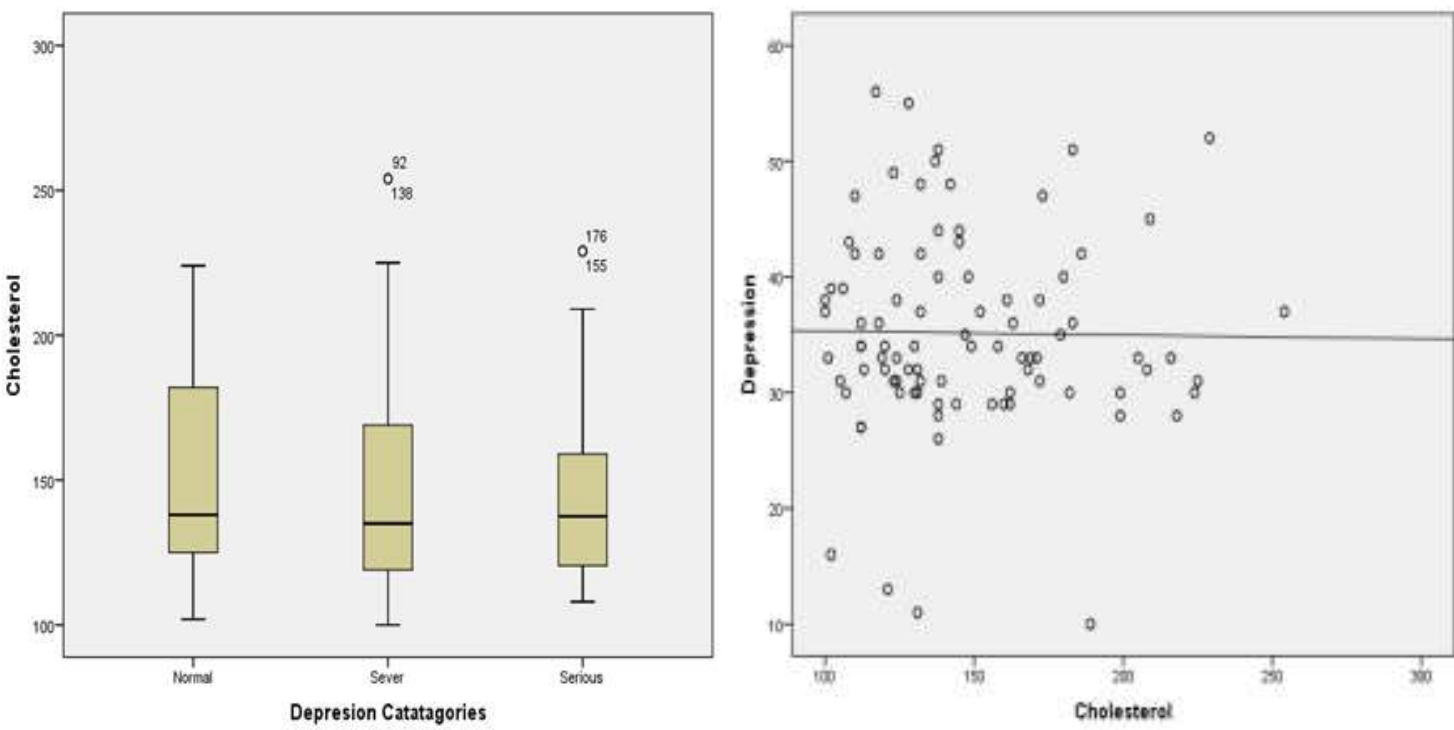

Figure 1. A: The level of mean serum cholesterol between attempters with different depression scores, B: Correlation between blood cholesterol level and depression scores

\section{Discussion}

Our results revealed that the cholesterol level was lower in the group with higher depression levels; however, there was no significant difference between the groups. There were significant differences between groups with different depression scores based on marital status, education, and hospitalization due to suicide, and the number of suicide attempts. Several studies have reported significant differences in suicide rates between males and females $(\mathbf{1}, 3,4,15)$. It has also been reported that differences exist in completed suicides and suicidal behaviors between males and females. For example, Segoviano et al. studied 261 patients with major depressive disorder (MDD), out of whom 59 had a history of suicide, and compared the results with another 206 individuals; they reported that low cholesterol levels were significantly associated with mood disorders and suicide. In the MDD group with a history of suicide, $49.2 \%$, and in the MDD group without a history of suicide, $38.2 \%$ had hypocholesterolemia. In the non-MDD healthy group, only $13.6 \%$ had hypocholesterolemia. In their study, the mean age in the group with a history of suicide was $35.2 \pm 10.5$ years, and most of them $(71.2 \%)$ were females (3).

In a study by Knowles et al. on 1,897 individuals, a significant association was observed between free cholesterol levels and suicide risk. In their study, in the group with a suicide history, a lower free cholesterol level was associated with a higher risk of suicide. The average age of the suicide attempters was 42.58 years, 
and females had a higher percentage of the attempters $(71.63 \%)(1)$.

In this study, the mean age of the total attempters was $26.39 \pm 10.75$ years., which is consistent with the results of some previous studies such as those conducted by $\mathrm{Wu}$ (15) and da Graça Cantarelli et al. (16). Their findings showed that suicide attempters were in a younger age range. We found no significant differences between the groups in terms of age, whereas some other studies have reported significant age differences between groups $(15,17)$.One study in Iran showed that there was no statistically significant relationship between age and sex in depressed patients with low cholesterol and triglycerides levels. The average depression score of the total population was $35.64 \pm 8.65$, indicating that most patients had moderate to high levels of depression. Similar to the study by Fiedorowicz et al. (4), we found higher suicide attempts in participants with higher depression scores; however, some other studies (18) have reported suicide attempts in patients with borderline depression disorders.

In the study by Fiedorowicz et al., contrary to their primary hypothesis, a higher level of cholesterol was found to be associated with an increased risk of suicide, instead of low cholesterol. In their study, the average age of suicide attempters was 33.4 years, and the majority of them were females. Thus, they reported that their results do not support the association between low cholesterol and subsequent suicide attempts (4). In the past, most of the cases of poisoning referred to hospitals were due to opioid abuse. However, nowadays, the trend of abuse has changed from opium to drugs (19). In this study, the majority of poisoned suicide attempters $(84 \%)$ hospitalized in the poisoning ward were drug users. This score was $86 \%$ in the study by Azizpour et al. conducted in 2016 (20). Since the last decade, several studies have reported that antidepressants reduce the risk of suicide among psychological patients (21), whereas others have demonstrated that the risk of suicide increases during antidepressant treatment (22). Recently, studies have indicated a possible increase in the risk of suicidal behavior among individuals taking psychiatric drugs (23). In $65 \%$ of individuals, benzodiazepine was the most commonly used agent for suicide. The results of the study by Geolaov et al. in England confirm our findings (24).

In this survey, although the mean depression intensity was 34.39 \pm 7.41 in males and 36.14 \pm 9.6 in females, with a higher percentage of severe depression in females in comparison to males ( $42.2 \%$ vs. $24.4 \%$ ), this measure had no significant association with the education level, marital status, economic status, and sex.

Depression, at the top of psychiatric disorders, and anxiety, anti-social personality disorder, substance abuse, and psychiatric comorbidity have been introduced as important predictors of suicide (25). In a survey in 2018, a family history of psychiatric disorders or committing suicide, having children, sex, age at the onset of depression, and drug abuse were associated with suicide attempts (26).

Different mechanisms have been proposed to explain the relationship between low cholesterol level and depression. For example, it has been suggested that low cholesterol is a consequence of depression, perhaps secondary to diminished appetite or weight loss (4). Wu et al. (15) indicated that a decrease in the level of cholesterol could reduce central serotonergic activity; moreover, cholesterol is a common causal factor for both cardiovascular and violent diseases. It has also been stated that cholesterol levels and mental state changes may be genetically correlated since some variations of chromosome 16 have been observed in association with both major depression and changes in the level of lecithin-cholesterol acyltransferase (LCAT) enzyme that mediates cholesterol esterification. Furthermore, cholesterol deficiency in neuronal membranes and dietary long-chain Poly Unsaturated Fatty Acid (PUFA) deficiencies can be related to the incidence of depression $(2,16)$.

Unlike some previous studies $(3,15)$, our results showed the lack of a significant association between lower serum cholesterol and suicide risk in this population. This difference can be due to the sample size, sample population composition and origin, the severity of depression in depressed individuals, and the lack of a control group. There are some studies $(4,17)$ that have yielded similar results in the same manner as ours, but they have detected no significant relationship between the level of cholesterol and suicide.

\section{Strengths and Limitations:}

This study had some limitations. First, it had a crosssectional design and some participants were not willing to participate in the survey. Despite this, attempts were made to resolve some problems by explaining the goals of the study. Second, individual differences and mental and psychological conditions could have affected the participants' answers, which was beyond our control. Third, although we controlled some confounders such as main demographic and clinical characteristics, serum cholesterol levels might have been influenced by additional characteristics such as nutritional condition and lifestyle patterns about which we could not collect any data.

\section{Conclusion}

Our results indicated that low total serum cholesterol may not be associated with depression; therefore, further and more comprehensive studies should be conducted to evaluate depression as a leading cause of suicide.

\section{Acknowledgment}

The authors thank the officials of the General Forensic Medicine Office of West Azerbaijan Province 
and the toxicology department of Taleghani University Hospital for their sincere collaboration. We are also grateful to Mrs. Khodaparst, who helped us collect the needed data.

\section{Ethical considerations}

Ethical issues (Including plagiarism, informed consent, misconduct, data fabrication and/or falsification, double publication and/or submission, redundancy, etc.) have been completely observed by the authors.

\section{Funding and support}

This research resulted from an independent research without receiving any financial support.

\section{Conflict of Interests}

The authors reported no conflict of interest.

\section{References}

1. Knowles EE, Curran JE, Meikle PJ, et al. Disentangling the genetic overlap between cholesterol and suicide risk. Neuropsychopharmacol. 2018;43(13):2556-63 [DOI:10.1038/s41386-018-0162-1]

2. Gvion Y, Apter A. Suicide and suicidal behavior. Public health reviews. 2012;34(2):9. [DOI:10.1007/BF03391677]

3. Segoviano-Mendoza M, Cárdenas-de la Cruz M, Salas-Pacheco J, et al. Hypocholesterolemia is an independent risk factor for depression disorder and suicide attempt in Northern Mexican population. BMC Psychiatr. 2018;18(1):7. [DOI:10.1186/s12888-018-1596-z]

4. Fiedorowicz JG, Coryell WH. Cholesterol and suicide attempts: a prospective study of depressed inpatients. Psychiatr Res. 2007;152(1):11-20. [DOI:10.1016/j.psychres.2006.09.003]

5. Shojaei A, Hashemi Nazari SS. Suicide mortality trends in four provinces of Iran with the highest mortality, from 2006-2016. J Res Health Sci. 2017;17(2): e00382.

6. Park S, Jang H. Correlations between suicide rates and the prevalence of suicide risk factors among Korean adolescents. Psychiatr Res. 2018;261:1437. [DOI:10.1016/i.psychres.2017.12.055]

7. Nazari H, Vahabzadeh D, Mohammadi SB, Zarei L, Ghahramani M. Social determinantfactors and their relationship with nutritional pattern in cardiovascular patients after hospital discharge. Maedica. 2016;11(3):214

8. Aghakhani N, Vahabzadeh D, Niroomand S, Asgarlii Z, Zaravar F. The relationship between spiritual wellbeing, anxiety and depression in old adults: A cross sectional study of Shiraz clinics, Iran. Middle East J Family Med. 2018;7(10):69.

9. Kupfer DJ, Regier DA. DSM 5 basics in: Diagnostic and statistical manual of mental disorders (DSM-5®): American Psychiatric Publication; 2013.

10. Moledina SM, Bhimji KM, Manji KP. Prevalence and associated factors of depression in an Asian community in Dar es Salaam, Tanzania. Psychiatr J. 2018;2018: 9548471 [DOI:10.1155/2018/9548471]

11. Strasser B, Sperner-Unterweger B, Fuchs D, Gostner JM. Mechanisms of inflammationassociated depression: immune influences on tryptophan and phenylalanine metabolisms. Curr Top Behav Neurosci . 2017;31:95-115. [DOI:10.1007/7854_2016_23]

12. Skibinska M, Kapelski P, Rajewska-Rager A, et al. Brain-derived neurotrophic factor (BDNF) serum level in women with first-episode depression, correlation with clinical and metabolic parameters. Nord J Psychiatr. 2018;72(3):191-6. [DOI:10.1080/08039488.2017.1415373]

13. Ho PS, Yen CH, Chen CY, Huang SY, Liang CS. Changes in cytokine and chemokine expression distinguish dysthymic disorder from major depression and healthy controls. Psychiatr Res. 2017;248:20-7. [DOI:10.1016/j.psychres.2016.12.014]

14. Grundy SM, Stone NJ, Bailey AL, et al. 2018 AHA/ACC/AACVPR/AAPA/ABC/ACPM/ADA/ AGS/APhA/ASPC/NLA/PCNA Guideline on the Management of Blood Cholesterol: Executive Summary: A Report of the American College of Cardiology/American Heart Association Task Force on Clinical Practice Guidelines. J Am College Cardiol. 2018:25708.

15. Wu S, Ding Y, Wu F, Xie G, Hou J, Mao P. Serum lipid levels and suicidality: a meta-analysis of 65 epidemiological studies. J Psychiatr Neurosci. 2016;41(1):56.

https://doi.org/10.1503/jpn.150079 [DOI:10.1503/ipn.150079.]

16. da Graça Cantarelli M, Nardin P, Buffon A, et al. Serum triglycerides, but not cholesterol or leptin, are decreased in suicide attempters with mood disorders. J Affect Disorder. 2015;172:403-9. [DOI:10.1016/i.jad.2014.10.033]

17. Bartoli F, Crocamo C, Dakanalis A, et al. Association between total serum cholesterol and suicide attempts in subjects with major depressive disorder: exploring the role of clinical and biochemical confounding factors. Clin Biochem. 2017;50(6):274-8 [DOI:10.1016/j.clinbiochem.2016.11.035]

Journal of Advances in Medical and Biomedical Research 
18. Atmaca M, Kuloglu M, Tezcan E, Gecici O, Ustundag B. Serum cholesterol and leptin levels in patients with borderline personality disorder. Neuropsychobiol. 2002;45(4):167-71. [DOI:10.1159/000063665]

19. R ahimi M, Lookzadeh S, Sadeghi R, et al. Predictive factors of mortality in acute amphetamine type stimulants poisoning; a review of 226 cases. Emergency. 2018;6(1):e-1 Epub 2018

20. Azizpour Y, Asadollahi K, Sayehmiri K, Kaikhavani S, Abangah G. Epidemiological survey of intentional poisoning suicide during 1993-2013 in Ilam Province, Iran. BMC Pub Health. 2016;16(1):902. [DOI:10.1186/s12889016-3585-9]

21. Martinez C, Rietbrock S, Wise L, et al. Antidepressant treatment and the risk of fatal and non-fatal self harm in first episode depression: nested case-control study. BMJ. 2005;330(7488):389. [DOI:10.1136/bmj.330.7488.389]

22. Hammad TA, Laughren T, Racoosin J. Suicidality in pediatric patients treated with antidepressant drugs. Arch General Psychiatr. 2006;63(3):332-9. [DOI:10.1001/archpsyc.63.3.332]

23. Pompili M, Giordano G, Lamis DA. Antidepressants and Suicide Risk: A Challenge. Understanding Suicide: Springer; 2016. p. 291 302. [DOI:10.1007/978-3-319-26282-6_24]

24. Geulayov G, Ferrey A, Casey D, Wells C, Fuller A, Bankhead C, et al. Relative toxicity of benzodiazepines and hypnotics commonly used for self-poisoning: An epidemiological study of fatal toxicity and case fatality. J Psychopharmacol. 2018;32(6):654-62 [DOI:10.1177/0269881118754734]

25. Gvion Y, Levi-Belz Y. Serious suicide attempts: systematic review of psychological risk factors. Front Psychiatr. 2018;9:56. [DOI:10.3389/fpsyt.2018.00056]

26. Pawlak JM1 MI, Dmitrzak-Węglarz M1, Szczepankiewicz A1,et al. Are suicide risk factors gender specific? Psychiatr Pol. 2018;52(1):21-32. [DOI:10.12740/PP/78974]

\section{How to Cite This Article:}

Aghakhani N, Boushehri B, Zarei A, Roshani R, Nazimi N, Cheraghi R, et al . Investigating the Relationship between Low Serum Cholesterol and Suicide in Attempters with Depression. J Adv Med Biomed Res. $2020 ; 28$ (131) :323-329

\section{Download citation:

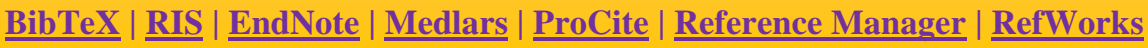

\section{Send citation to:}

9. Mendeley 2 Zotero Prirki k. RefWorks 